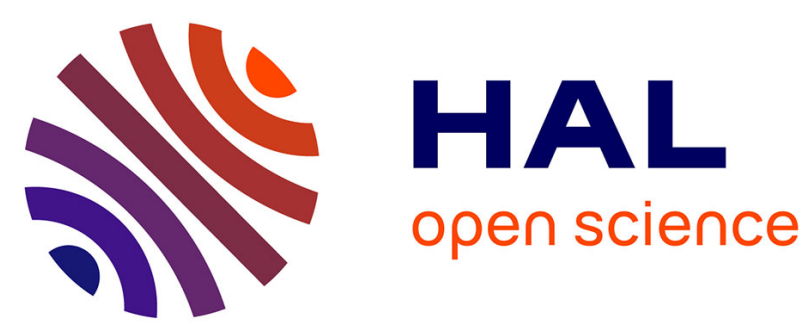

\title{
Spatial variability of the 12-lead surface ECG as a tool for noninvasive prediction of catheter ablation outcome in persistent atrial fibrillation
}

Marianna Meo, Vicente Zarzoso, Olivier Meste, Decebal Gabriel Latcu, Nadir Saoudi

\section{To cite this version:}

Marianna Meo, Vicente Zarzoso, Olivier Meste, Decebal Gabriel Latcu, Nadir Saoudi. Spatial variability of the 12-lead surface ECG as a tool for noninvasive prediction of catheter ablation outcome in persistent atrial fibrillation. IEEE Transactions on Biomedical Engineering, 2013, 60 (1), pp.20-27. 10.1109/TBME.2012.2220639 . hal-00862201

\section{HAL Id: hal-00862201 https://hal.science/hal-00862201}

Submitted on 16 Sep 2013

HAL is a multi-disciplinary open access archive for the deposit and dissemination of scientific research documents, whether they are published or not. The documents may come from teaching and research institutions in France or abroad, or from public or private research centers.
L'archive ouverte pluridisciplinaire HAL, est destinée au dépôt et à la diffusion de documents scientifiques de niveau recherche, publiés ou non, émanant des établissements d'enseignement et de recherche français ou étrangers, des laboratoires publics ou privés. 


\title{
Spatial Variability of the 12-Lead Surface ECG as a Tool for Noninvasive Prediction of Catheter Ablation Outcome in Persistent Atrial Fibrillation
}

\author{
Marianna $\mathrm{Meo}^{1}$, Student Member, IEEE, Vicente Zarzoso ${ }^{1}$, Senior Member, IEEE, Olivier Meste ${ }^{1}$, Member, IEEE, \\ Decebal Gabriel Latcu ${ }^{2}$, and Nadir Saoudi ${ }^{2}$
}

\begin{abstract}
Atrial fibrillation (AF) is the most common sustained cardiac arrhythmia encountered in clinical practice. Radiofrequency catheter ablation (CA) is increasingly employed to treat this disease, yet selection of persistent AF patients who will benefit from this treatment remains a challenging task. Several parameters of the surface electrocardiogram (ECG) have been analyzed in previous works to predict AF termination by $\mathrm{CA}$, such as fibrillatory wave (f-wave) amplitude. However, they are usually manually computed and only a subset of electrodes is inspected. In the present study, a novel perspective of the role of f-wave amplitude as a potential non-invasive predictor of CA outcome is adopted by exploring ECG inter-lead spatial variability. An automatic procedure for atrial amplitude computation based on cubic Hermite interpolation is first proposed. To describe the global f-wave peak-to-peak amplitude distribution, signal contributions from multiple leads are then combined by condensing the most representative features of the atrial signal in a reduced-rank approximation based on principal component analysis (PCA). We show that exploiting ECG spatial diversity by means of this PCA-based multilead approach does not only increase the robustness to electrode selection, but also substantially improves the predictive power of the amplitude parameter.
\end{abstract}

Index Terms-Atrial fibrillation (AF), catheter ablation (CA), principal component analysis (PCA), fibrillatory wave (f-wave).

\section{INTRODUCTION}

D espite major advances in its treatment, atrial fibrillation (AF) remains a significant cause of cardiovascular morbidity and mortality, especially those arising from stroke and heart failure. AF is characterized by uncoordinated atrial activation with consequent loss of atrial mechanical function. Various therapies have been used, such as electrical cardioversion, antiarrhythmic drugs and catheter ablation (CA) techniques. However, current AF management guidelines provide no systematic treatment recommendations, since the various mechanisms and patterns of this disease are not completely understood yet [1]. The CA therapy has been increasingly

Manuscript received April 18, 2012; revised July 12, 2012, and accepted August 20, 2012. This work is partly supported by the French National Research Agency under contract ANR-2010-JCJC-0303-01 "PERSIST". Marianna Meo is funded by a doctoral grant from the French Ministry of Higher Education and Research.

${ }^{1}$ Marianna Meo, Vicente Zarzoso and Olivier Meste are with the Laboratoire d'Informatique, Signaux et Systèmes de Sophia Antipolis (I3S), Université Nice Sophia Antipolis, CNRS, France (e-mail:\{meo, zarzoso, meste\}@i3s.unice.fr).

${ }^{2}$ Decebal G. Latcu and Nadir Saoudi are with the Service de Cardiologie, Centre Hospitalier Princesse Grace, Monaco (e-mail: \{dg.latcu, nsaoudi\}@ chpg.mc). used as a first-line option in recent years [2]. Nowadays, the stepwise technique is the most widespread. It consists in a sequence of actions aiming at suppressing the sources of abnormal rhythm by radiofrequency cauterization, typically including pulmonary vein (PV) isolation with an endpoint of lasso-proven disconnection [3], [4] and ablation of complex fractionated atrial electrograms (CFAE) [5]. However, CA of persistent AF is a lengthy and expensive procedure, and inconsistent success rates have been reported by different centers practicing the therapy. Consequently, this has called for adequate tools exploiting information about AF electrophysiology to perform an a priori selection of patients who are more likely to positively respond to CA therapy.

$\mathrm{AF}$ analysis by means of non-invasive ECG recordings has received considerable attention in recent years, spurring the development of signal processing techniques for a more advanced characterization of the atrial waveforms. So far, the cycle length or inverse dominant frequency (repetition rate) of the atrial waves is one of the most studied parameters. Its clinical significance is founded on its ability to identify pathophysiological mechanisms and predict therapy efficacy [6]. Other studies [7] underline that higher pre-procedural values of the amplitude of the fibrillatory waves (f-waves) can be associated with a higher probability of successful procedures. Nevertheless, several limitations affect the discriminative power of such classifiers. First and foremost, their manual acquisition considerably increases error probability, as it is influenced by operator's subjectivity. Furthermore, the single-lead perspective neglects potentially useful information from other leads. Yet considering multiple leads may help shed some light on the disease by analyzing the heart electrical activity from different spatial locations.

In this framework, our research aims at the noninvasive prediction of CA outcome by characterizing the f-wave amplitude variability across the multiple leads of the ECG. An automatic algorithm for computing atrial amplitude on a single lead is first proposed. F-wave amplitude is quantified by interpolating local extrema points of the actual signal. This procedure represents the starting point of a multilead algorithm, which yields a parameter describing f-wave amplitude spatial distribution over the eight independent ECG leads by applying principal component analysis (PCA). Our investigation demonstrates the benefits of exploiting ECG spatial diversity, which enhances the f-wave amplitude discriminative power and improves its robustness to electrode selection in CA outcome prediction. 


\section{Methods}

\section{A. ECG Data and Acquisition System}

The 28 patients involved in this study were all affected by persistent $\mathrm{AF}$ and underwent $\mathrm{CA}$. They all gave their informed consent. The procedure was performed with the aid of Prucka Cardiolab and Biosense CARTO electrophysiology measurement systems at the Cardiology Department, Princess Grace Hospital, Monaco. For each patient, one-minute 12lead surface ECG was recorded at a sampling rate of $1 \mathrm{kHz}$ at the beginning of the ablation procedure. An example on lead $V_{1}$ is illustrated in Fig. 1. Ablation was performed in a stepwise manner [2], starting with circumferential PV ablation with LASSO-guided disconnection, followed by fragmented potentials, non-PV triggers, roof line and mitral isthmus line right atrial ablation.

The present study adopts a short-term definition of procedural outcome, providing a preliminary perspective of immediate $\mathrm{CA}$ effectiveness and examining the risk of $\mathrm{AF}$ recurrence in the very first postoperative period. Short-term success is defined as AF conversion either directly to sinus rhythm or intermediate tachyarrhythmia, exclusively by ablation or by CA followed by electrical cardioversion. Three patients who did not experience AF termination after a first procedure underwent a second ablation, making a total of $n_{P}=31$ procedures. Accordingly, $n_{S}=26$ procedures were successful, whereas $n_{F}=5$ procedures failed.

\section{B. ECG Preprocessing and Atrial Activity Extraction}

The preprocessing steps described in the sequel are motivated by our interest in examining f-wave amplitude properties, regardless of their temporal location or shape factors. Indeed, focusing on amplitude parameters allows us to avoid frequency-based methods requiring atrial activity (AA) extraction, widely regarded as a not trivial task, thus considerably simplifying our approach. Our strategy also avoids straightforward analysis of ECG morphology, which is reasonable to apply when dealing with f-waves whose shape is very repetitive and easy to segment; this is typically not the case in more advanced forms of the disease such as the persistent AF examined in the present study. Moreover, fwave amplitude predictive power is widely recognized in the framework of persistent AF ablation, as it represents the sum of local depolarization phenomena involving cardiac cells [7]. Its examination proves to be easier to perform and equally efficient compared to morphology-based analysis, requiring more complex heart models [8], [9]. Finally, to our knowledge, no correlation between f-wave morphology and CA outcome has been shown to date.

Accordingly, ECG recordings are first processed by a fourthorder zero-phase type II Chebyshev bandpass filter with -3 $\mathrm{dB}$ attenuation at $0.5 \mathrm{~Hz}$ and $30 \mathrm{~Hz}$ cut-off frequencies. The filter selected can accurately reduce the influence of noisy components typically encountered in ECG analysis without deforming signal shape [10], [11]. The choice of pass-band is meant to exclusively enhance $\mathrm{AF}$ content, whose dominant frequency ranges between 3 and $12 \mathrm{~Hz}$, by suppressing baseline wandering and high frequency noise such as myoelectric

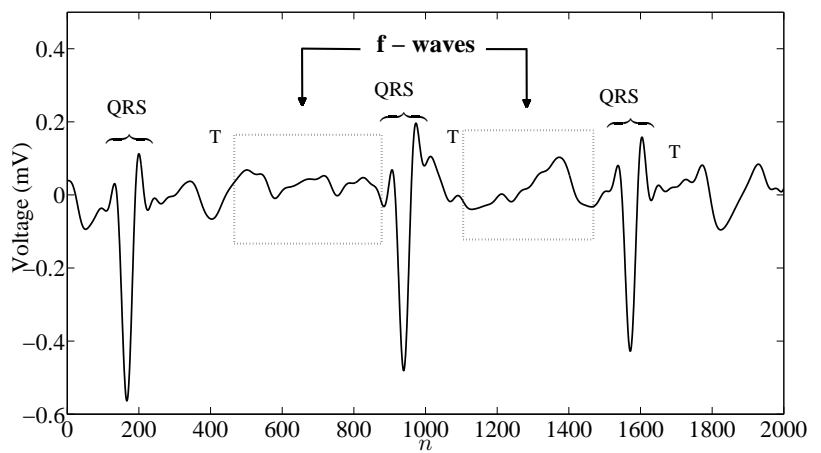

Fig. 1: Example of ECG recording during AF and its characteristic waves. Boxes highlight TQ intervals which are concatenated to form the AA signal $\mathbf{Y}_{\mathrm{AA}}$ in eqn. (1).

artifacts and $50 \mathrm{~Hz}$ power line interference. Automatic detection of ECG fiducial points is subsequently performed so as to properly segment the TQ intervals. $\mathrm{R}$ wave time instants are retrieved on lead $\mathrm{V}_{1}$ by applying the Pan-Tompkins' algorithm [12], whereas $\mathrm{Q}$ wave onset and $\mathrm{T}$ wave offset are detected with an improved version of Woody's method [13]. Mean-centering and concatenation of such segments are finally accomplished, thereby yielding an $(L \times N)$ data matrix $\mathbf{Y}_{\mathrm{AA}}$ representing atrial activity (AA) only:

$$
\mathbf{Y}_{\mathrm{AA}}=\left[\mathbf{y}_{\mathrm{AA}}(1) \cdots \mathbf{y}_{\mathrm{AA}}(N)\right] \in \mathbb{R}^{L \times N}
$$

where vector $\mathbf{y}_{\mathrm{AA}}(n)=\left[y_{1}(n), \ldots, y_{L}(n)\right]^{\mathrm{T}}$ stands for the multilead AA signal at sample index $n, L$ is the number of leads used, and $N$ the number of samples of the AA signal $y_{\ell}(n)$ for each lead $\ell=1,2, \ldots, L$. All residual artifacts and spurious peaks due to concatenation have been automatically removed by means of signal first-derivative thresholding.

Unlike previous works, a subset of all leads of the standard ECG has been used in our algorithms. Indeed, Einthoven's standard leads (or limb leads, I, II, III) and Golbderger's augmented leads (aVR, aVL, aVF) are derived from the same three measurement points, and are thus redundant (linearly related) [2]. Accordingly, lead III has been discarded by our analysis, as I and II are sufficient to characterize heart electrical activity on the frontal plane. Finally, all precordial leads have been introduced too, in order to record the cardiac electric potential in a cross sectional plane, for a total of $L=8$ leads, that is, I, II, $\mathrm{V}_{1}-\mathrm{V}_{6}$.

\section{Automatic F-wave Amplitude Computation}

According to previous studies, successful CA procedures can be predicted by higher values of f-wave peak-to-peak amplitude on the surface ECG [7]. Nevertheless, prediction accuracy is so far affected by amplitude manual computation, leading to higher error probability, especially in the presence of irregular and complex waveform patterns. Similar shortcomings can occur when dealing with different operators performing the acquisition of such a parameter, so its measure is not uniquely defined. Given these conditions, we develop an automatic procedure which computes the amplitude value of the AA signal $y_{\ell}(n)$ on a specific lead $\ell=1,2, \ldots, L$. It 


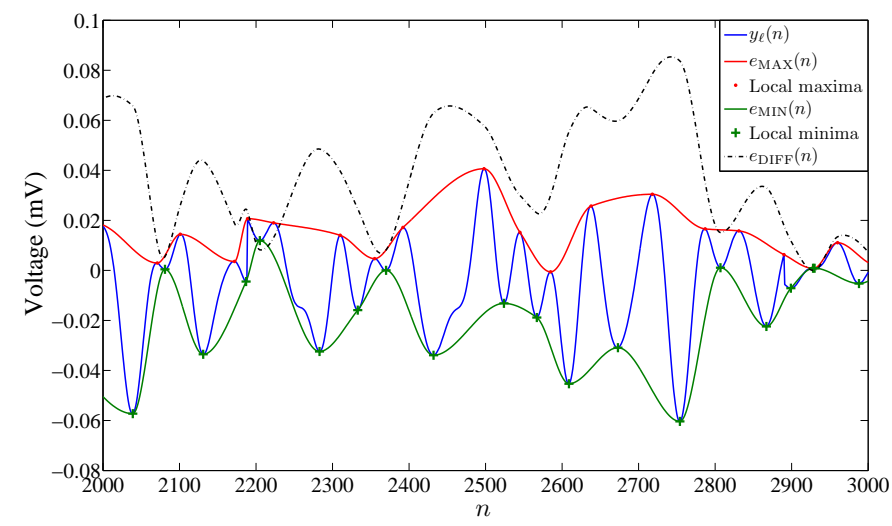

Fig. 2: Single-lead atrial waveform interpolation algorithm. Upper and lower envelopes $e_{\mathrm{MAX}}$ and $e_{\mathrm{MIN}}$ pass through the local extrema of the input signal $y_{\ell}$; their subtraction yields $e_{\mathrm{DIFF}}$.

starts from the detection of the local maxima of $y_{\ell}(n)$ and the estimation of an upper envelope $e_{\operatorname{MAX}}(n)$ by the shapepreserving piecewise cubic Hermite interpolating polynomial (PCHIP) [14]. Similarly, a lower envelope $e_{\mathrm{MIN}}(n)$ passing through the minima is obtained as well. This strategy of automatic detection of local extrema of the f-waves proves more efficient than the manual approach, as it mainly focuses on the global trend of the curve. In addition, PCHIP interpolation reduces the negative effect of local spurious peaks and signal artifacts. Peaks located near the edge between two consecutive TQ intervals are not taken into account in the interpolation. The difference $e_{\mathrm{DIFF}}(n)=\left|e_{\mathrm{MAX}}(n)-e_{\mathrm{MIN}}(n)\right|$ between the two curves outlines the general trend of the main oscillations of $\mathrm{f}$-waves, and its temporal mean over the AA signal length $N$ :

$$
D(y)=\frac{1}{N} \sum_{n=1}^{N} e_{\mathrm{DIFF}}(n)
$$

represents the final output of our algorithm. The operator $D(\cdot)$ is characterized by an offset-invariance property:

$$
D(k y+\alpha)=|k| D(y), \quad \forall k, \alpha \in \mathbb{R} .
$$

Index $D$ is capable of summarizing the information about $\mathrm{f}$ wave over the whole recording and its peak-to-peak amplitude pattern in a single objective parameter computed in a fully automated manner.

\section{Extension to Multilead Recordings}

A further limitation of previous methods is that f-wave amplitude is examined on individual leads only (e.g., $\mathrm{V}_{1}$ or II) [7], without fully exploiting the spatial diversity typical of multilead ECG recordings. It is widely known that the largest atrial-to-ventricular amplitude ratio can be found on lead $V_{1}$ [15]. Yet its proximity to the right atrial free wall may neglect significant information about other sites, for instance, the left atrium and the PVs, which play a crucial role in $\mathrm{AF}$ initiation and maintenance [3]. Indeed, not only these approaches do not rely on contributions provided by other ECG leads and their mutual relations, but selection of single electrodes is not driven by further systematic criteria.
These conditions represent the basis for the development of an extension of the algorithm described in Sec. II-C so as to broaden single-lead characterization of f-wave amplitude by considering more than one lead. The rationale of our hypothesis is the intrinsic variability of the surface ECG across leads, as they are all measured from different points of the patient's body. Our multivariate AA signal can be regarded as a surface mixture of a certain number of unknown sources. This assumption motivates the application of principal component analysis (PCA). PCA can express our multilead recordings as a function of these uncorrelated, most representative sources, or principal components (PCs) [16], by retaining the maximum amount of information as measured by variance. PCs are computed and ordered so that the first few retain most of the variation present in all of the original signals. Since variance and amplitude are closely related, the most dominant PCs are expected to provide an accurate description of the spatial distribution of AA amplitude.

According to PCA, our multivariate observation matrix $\mathbf{Y}_{\mathrm{AA}}$ can be decomposed as the linear model:

$$
\mathbf{y}_{\mathrm{AA}}(n)=\mathbf{M x}(n)=\sum_{k=1}^{L} \mathbf{m}_{k} x_{k}(n)
$$

where $\mathbf{M}$ is the mixing matrix with mutually orthogonal columns that relates the sources $x_{k}(n), k=1, \ldots, L$ with the multilead observations $\mathbf{y}_{\mathrm{AA}}(n)$. The derivation of PCs is based on the assumption that $\mathbf{y}_{\mathrm{AA}}(n)$ is a zero-mean random process. The entries of column vector $\mathbf{m}_{k}$ weigh the contribution of the corresponding PC $x_{k}$ to the leads in $\mathbf{y}_{\mathrm{AA}}(n)$, and thus render the principal propagation direction or spatial topography of each source [17]. This decomposition effectively exploits spatial relationships among ECG leads and discards redundant information by keeping only the most significant PCs and their corresponding directions.

\section{E. Reduced-Rank PCA for Atrial Signal Characterization}

Knowing that the dominant PC retains the highest percentage of the AA signal variance, it seems reasonable to attempt the exploitation of this property to extract the most significant information from the AA signal. It is worth introducing the general concept of spatial filtering, as an optimal tool for recovering a certain signal of interest from the ensemble of observations [18]. In the PCA framework, the idea is to look for a linear function of the elements of $\mathbf{y}_{\mathrm{AA}}$ having maximum variance. In line with this rationale, the spatial filter $\mathbf{m}_{1}$ is the linear weight vector yielding the dominant PC $x_{1}$ as

$$
x_{1}(n)=\mathbf{m}_{1}^{\mathrm{T}} \mathbf{y}_{\mathrm{AA}}(n),
$$

thus isolating the contribution of $x_{1}$ to $\mathbf{y}_{\mathrm{AA}}$ from that coming from other sources. The first principal direction $\mathbf{m}_{1}$ maximizes the variance of $x_{1}$, given by $\mathrm{E}\left[x_{1}^{2}\right]=\mathbf{m}_{1}^{\mathrm{T}} \mathbf{R}_{\mathrm{y}} \mathbf{m}_{1}$, under the constraint $\left\|\mathbf{m}_{1}\right\|=1$, where $\mathbf{R}_{\mathrm{y}}$ denotes the covariance matrix of the observations. The maximal variance is achieved when $\mathbf{m}_{1}$ is the normalized dominant eigenvector (related to the highest eigenvalue) of $\mathbf{R}_{\mathrm{y}}$. The output function in eqn. (5) can clearly be seen as a weighted average of the observed random 
variables. The remaining principal directions are also estimated by variance maximization, but including orthogonality constraints with respect to the previously obtained directions.

The truncation of eqn. (4) yields the rank- $R$ approximation:

$$
\hat{\mathbf{y}}_{\mathrm{AA}}(n)=\sum_{k=1}^{R} \mathbf{m}_{k} x_{k}(n)
$$

with $R<L$. Note that $\hat{\mathbf{y}}_{\mathrm{AA}}$ has the same size as $\mathbf{y}_{\mathrm{AA}}$. In this work, we propose to approximate the AA observations by exclusively emphasizing the influence of the first $\mathrm{PC}$ on the selected leads; this is achieved with $R=1$ in eqn. (6). This rank-1 reconstruction allows not only the enhancement of the most descriptive component in terms of variance but, as shown in Sec. III, also the suppression of irrelevant and/or noisy elements which can pollute signal content.

\section{F. Multilead F-wave Amplitude Descriptor}

Once AA signal approximation is carried out, the algorithm presented in Sec. II-C is run on each lead. Unlike eqn. (2), which returns a scalar value as output, we obtain now an $L$ component vector $D_{L}$, whose entries represent the temporal average of peak-to-peak amplitude envelope values on every electrode:

$$
\mathbf{D}_{L}=\left[d_{1}, d_{2}, \ldots, d_{L}\right]^{\mathrm{T}} .
$$

where $d_{\ell}=D\left(m_{\ell 1} x_{1}\right)=\left|m_{\ell 1}\right| D\left(x_{1}\right)$, according to the property in eqn. (3), and $m_{\ell 1}=\left[\mathbf{m}_{1}\right]_{\ell}$ is the $\ell$ th component of the principal direction $\mathbf{m}_{1}$. The entries of $\mathbf{D}_{L}$ are then sorted in increasing order and their median value $\widetilde{D}_{L}$ is finally obtained. The choice of the median is justified by its ability to describe overall data distribution without loss of generality, with a higher degree of robustness to outliers, compared to other statistical descriptors such as the mean value. Parameter $\widetilde{D}_{L}$ can be considered as a descriptor of the global spatial distribution of f-wave amplitudes over the observed leads.

\section{G. Statistical Analysis}

According to the protocol explained in Sec. II-A, ablation outcome categories to be discriminated are referred to as "AF termination" and "non AF termination" by CA. All parameters are expressed as mean \pm standard deviation in Table I. Once data Gaussianity has been verified through the Lilliefors' test, differences between the two classes are statistically assessed by an unpaired Student's $t$-test if data follow a normal distribution, a two-sample Wilcoxon rank sum test otherwise, under a confidence level $\alpha=0.05 ; p$ values associated with each unpaired test are reported in Table I as well. The quality of the binary classification model is measured by the area under curve (AUC) of its receiver operating characteristic (ROC) curve, based on the maximization of sensitivity and specificity, i.e., the rate of true positives and true negatives, respectively. A leave-one-out (LOO) cross-validation technique is employed to assess the generalization ability of our analysis to an independent dataset. More specifically, AUC values are computed several times by keeping a sample of 30 procedures out of 31 and thus discarding one subject at each iteration, so as to compute their average value at the final step. AUC values of
TABLE I: Interclass statistical analysis

\begin{tabular}{|c||c|c|c|}
\hline & $\begin{array}{c}\text { AF } \\
\text { termination }\end{array}$ & $\begin{array}{c}\text { Non AF } \\
\text { termination }\end{array}$ & $p$-value \\
\hline$\widetilde{D}_{8}$ & $0.038 \pm 0.019$ & $0.015 \pm 0.007$ & $9.56 \cdot 10^{-4}$ \\
\hline$\widetilde{D}_{12}$ & $0.030 \pm 0.012$ & $0.015 \pm 0.006$ & $8 \cdot 10^{-3}$ \\
\hline $\bar{D}_{8}$ & $0.042 \pm 0.023$ & $0.022 \pm 0.01$ & $6.4 \cdot 10^{-2}$ \\
\hline $\bar{D}_{12}$ & $0.049 \pm 0.070$ & $0.022 \pm 0.01$ & $5.0 \cdot 10^{-2}$ \\
\hline$D\left(V_{1}\right)$ & $0.068 \pm 0.022$ & $0.054 \pm 0.017$ & $1.85 \cdot 10^{-1}$ \\
\hline $\mathrm{RMS}\left(V_{1}\right)$ & $0.075 \pm 0.110$ & $0.027 \pm 0.017$ & $1.55 \cdot 10^{-1}$ \\
\hline SampEn( $\left.L_{s}, r_{s}^{(1)}\right)$ & $0.299 \pm 0.063$ & $0.218 \pm 0.107$ & $2.67 \cdot 10^{-1}$ \\
\hline SampEn( $\left(L_{s}, r_{s}^{(2)}\right)$ & $0.143 \pm 0.029$ & $0.106 \pm 0.052$ & $3.25 \cdot 10^{-1}$ \\
\hline$\widetilde{R M S}_{8}$ & $0.016 \pm 0.009$ & $0.009 \pm 0.006$ & $1.40 \cdot 10^{-1}$ \\
\hline$\widehat{R M S}_{12}$ & $0.024 \pm 0.015$ & $0.016 \pm 0.008$ & $1.40 \cdot 10^{-1}$ \\
\hline$\overline{\mathrm{RMS}}_{8}$ & $0.021 \pm 0.016$ & $0.021 \pm 0.021$ & $3.76 \cdot 10^{-1}$ \\
\hline$\overline{\mathrm{RMS}}_{12}$ & $0.037 \pm 0.048$ & $0.026 \pm 0.024$ & $2.48 \cdot 10^{-1}$ \\
\hline
\end{tabular}

each descriptor are displayed in Table II; in addition, we report the corresponding optimal cut-off points, associated with the maximization of the sum of true positive and true negative cases determined over the 31-procedure database.

\section{RESUlTS}

To test the single-lead algorithm presented in Sec. II-C, parameter $D$ in eqn. (2) has been computed on lead $\mathrm{V}_{1}$, yielding $D\left(\mathrm{~V}_{1}\right)$. Another classical single-lead method focusing on AA signal magnitude has been considered for the sake of comparison, namely, the root mean square value on the lead $\mathrm{V}_{1}$, denoted $\mathrm{RMS}\left(\mathrm{V}_{1}\right)$. Finally, a parallel with a non-linear complexity index, the sample entropy SampEn [19], has been drawn as well on the same electrode. This feature depends on two parameters: $L_{s}$ and $r_{s}$. Parameter $L_{s}$ stands for the length of the sequences the ECG recording is split in. Such sequences are then compared, and the tolerance for accepting matches is represented by the parameter $r_{s}$. This parameter is chosen as a fraction of the AA input signal standard deviation on $\mathrm{V}_{1}$, denoted $\sigma_{V_{1}}$, so as to assure the translation and scale invariance of SampEn. Both parameters have been tuned according to the guidelines given in [20], yielding $L_{s}=2$ besides two values of $r_{s}$, namely, $r_{s}^{(1)}=0.1 \sigma_{V_{1}}$ and $r_{s}^{(2)}=0.2 \sigma_{V_{1}}$.

In the multilead framework, the discriminative power of our descriptor $\widetilde{D}_{L}$ has been assessed both on the ensemble of eight linearly independent ECG leads $(L=8)$, as defined in Sec. II-A, and on the full standard ECG $(L=12)$, giving rise to indices $\widetilde{D}_{8}$ and $\widetilde{D}_{12}$, respectively. The same analysis has been led by using the mean instead of the median when averaging the entries of vector $\mathbf{D}_{L}$ in eqn. (7), yielding $\bar{D}_{8}$ and $\bar{D}_{12}$. This is the index proposed in [21], but using PCHIP instead of cubic spline interpolation. Finally, the ability of PCHIP interpolation to effectively approximate the AA signal general trend and give a measure of f-wave peak-to-peak amplitude is also examined. For sake of comparison with our algorithm, after PCA application and rank-1 truncation explained in Sec. II-E, the RMS value is computed on every row of the data matrix $\hat{\mathbf{Y}}_{\mathrm{AA}}$ resulting from eqn. (6). Both sets of ECG leads have been considered for the computation of this feature as well, from which the median $\left(\widetilde{\mathrm{RMS}}_{8}, \widetilde{\mathrm{RMS}}_{12}\right)$ and mean values $\left(\overline{\mathrm{RMS}}_{8}, \overline{\mathrm{RMS}}_{12}\right)$ have been determined.

Robustness of our multilead predictor $\widetilde{D}_{L}$ to the choice of ECG leads has also been tested. For each value of lead-subset 
TABLE II: CA outcome prediction performance

\begin{tabular}{|c||c|c|}
\hline & AUC & Best cut-off \\
\hline$\widetilde{D}_{8}$ & 0.98 & 0.023 \\
\hline$\widetilde{D}_{12}$ & 0.91 & 0.022 \\
\hline $\bar{D}_{8}$ & 0.77 & 0.027 \\
\hline $\bar{D}_{12}$ & 0.78 & 0.027 \\
\hline$D\left(V_{1}\right)$ & 0.68 & 0.060 \\
\hline $\mathrm{RMS}\left(V_{1}\right)$ & 0.71 & 0.013 \\
\hline SampEn $\left(L_{s}, r_{s}^{(1)}\right)$ & 0.75 & 0.289 \\
\hline SampEn $\left(L_{s}, r_{s}^{(2)}\right)$ & 0.72 & 0.139 \\
\hline$\widetilde{R M S}_{8}$ & 0.72 & 0.005 \\
\hline$\widetilde{R M S}_{12}$ & 0.72 & 0.011 \\
\hline$\overline{R M S}_{8}$ & 0.63 & 0.012 \\
\hline$\overline{R M S}_{12}$ & 0.67 & 0.014 \\
\hline
\end{tabular}

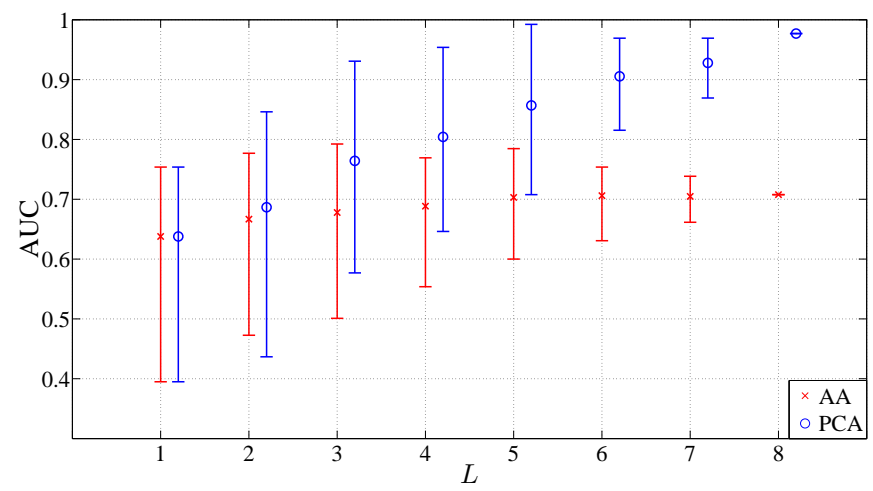

Fig. 3: $\widetilde{D}_{L}$ prediction performance as a function of the size $L$ of the subset of the 8 independent ECG leads. Vertical lines stretch between the minimum and maximum AUC values obtained for each $L$; mean values are shown with markers. AA: measuring $\widetilde{D}_{L}$ directly from the observed AA signal. PCA: measuring $\widetilde{D}_{L}$ from approximation (6) with $R=1$.

size $L$ ranging from 1 up to 8 , the proposed multilead predictor has been run on all $8 ! /((8-L) ! L !)$ possible lead combinations. CA outcome prediction performance has then been assessed for each lead combination from the corresponding values of $\widetilde{D}_{L}$, using the LOO technique. In this manner, the minimum, maximum and mean AUC values over all $L$-lead subset combinations have been obtained as a function of the subset dimension $L$; their corresponding intervals are displayed in Fig. 3. The lead combinations providing the best prediction performance for each subset dimension are shown in Table III. Likewise, the straightforward application of the algorithm described in Sec. II-C on the AA signal (namely, without PCA approximation) has been tested, in order to demonstrate PCA effectiveness in filtering and enhancing content-bearing information from the AA signal. Along the same lines, PCA's ability to compress data into few, most representative PCs is shown in Fig. 4, in which approximation quality is assessed by the AUC criterion; classification performance over all ECG leads is evaluated as a function of $R$, the number of terms retained in the truncation in eqn. (6), ranging from 1 (the value adopted in our algorithm) to 8 (full-rank reconstruction of the input data). This comparison is validated by the LOO procedure.
TABLE III: ECG lead subsets with optimal prediction performance based on parameter $\widetilde{D}_{L}$

\begin{tabular}{|c|c|}
\hline Number of leads $(L)$ & Leads \\
\hline 1 & $\mathrm{~V}_{3}$ \\
\hline 2 & II, $\mathrm{V}_{4}$ \\
\hline 3 & I, $\mathrm{V}_{3}, \mathrm{~V}_{4}$ \\
\hline 4 & II, $\mathrm{V}_{3}, \mathrm{~V}_{4}, \mathrm{~V}_{5}$ \\
\hline 5 & II, $\mathrm{V}_{1}, \mathrm{~V}_{3}, \mathrm{~V}_{5}, \mathrm{~V}_{6}$ \\
\hline \multirow{2}{*}{6} & {$\left[\mathrm{I}, \mathrm{II}, \mathrm{V}_{1}, \mathrm{~V}_{3}, \mathrm{~V}_{5}, \mathrm{~V}_{6}\right]$} \\
& {$\left[\mathrm{I}, \mathrm{II}, \mathrm{V}_{2}, \mathrm{~V}_{4}, \mathrm{~V}_{5}, \mathrm{~V}_{6}\right]$} \\
& {$\left[\mathrm{II}, \mathrm{V}_{1}, \mathrm{~V}_{2}, \mathrm{~V}_{4}, \mathrm{~V}_{5}, \mathrm{~V}_{6}\right]$} \\
\hline \multirow{2}{*}{7} & {$\left[\mathrm{II}, \mathrm{V}_{1}, \mathrm{~V}_{2}, \mathrm{~V}_{4}, \mathrm{~V}_{5}, \mathrm{~V}_{6}\right]$} \\
& {$\left[\mathrm{II}, \mathrm{V}_{1}, \mathrm{~V}_{2}, \mathrm{~V}_{3}, \mathrm{~V}_{4}, \mathrm{~V}_{5}, \mathrm{~V}_{6}\right]$} \\
\hline
\end{tabular}

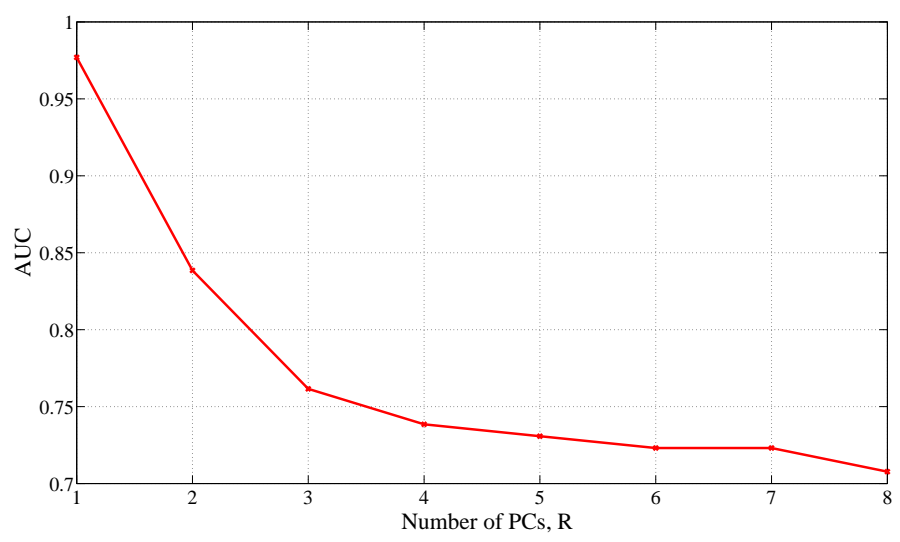

Fig. 4: Classification performance for the 8 linearly independent ECG leads. AUC of $\widetilde{D}_{8}$ as a function of $R$, the number of PCs retained in approximation (6).

\section{DISCUSSION}

\section{A. Manual vs. Automatic Computation of Atrial Amplitude}

The predictive value of f-wave amplitude for CA of persistent AF has been scrutinized in previous studies [7]. However, not only it has been manually obtained, but only one ECG lead has been considered in its computation. By contrast, a method aiming at automatically computing f-wave peak-topeak amplitude on a single lead is proposed in our study (Sec. II-C). Direct correlation with procedural AF termination, as demonstrated in [7] for the manual amplitude index, is confirmed for its automatic counterpart proposed herein. This is shown by the results of the single-lead parameters in Table I, namely $D\left(V_{1}\right)$ and $\operatorname{RMS}\left(\mathrm{V}_{1}\right)$. However, the low $p$ values obtained with these indices prove their weak discriminating ability in the dataset under examination.

From the analysis of f-wave peak-to-peak amplitude it can be inferred that our single-lead automatic approach, yielding $D\left(\mathrm{~V}_{1}\right)$, returns values close to those manually computed in [7] $(0.08 \pm 0.03 \mathrm{mV}$ for successful CA procedures, $0.05 \pm 0.03 \mathrm{mV}$ for the failing ones, $p<0.01$, AUC $=0.77$ ). This comparison only aims at underlining the ability of our procedure to automatically reproduce such results and associate higher amplitude values with successful CA procedures, event though the respective databases are not directly comparable and interclass statistically significant differences are not verified on the signals we examined. 


\section{B. Comparison with Other Atrial Signal Parameters}

Another parameter considered in our investigation is the RMS value, whose prediction performance proves rather inaccurate. Furthermore, the unpaired test returns non-significant differences between the classes considered. Concerning the sample entropy SampEn, despite the fairly good AUC values and the presence of statistically significant differences between the categories of interest, $\mathrm{AF}$ termination by $\mathrm{CA}$ is not predicted by low SampEn values as in [22] for electrical cardioversion. This is in contrast with the assumption of a positive correlation between the sample entropy and AA signal spatio-temporal complexity. One would actually expect that more organized AA waveforms as measured by lower SampEn values render a less critical disease profile, easier to be treated by CA. Yet this hypothesis is not verified in our study, regardless of the threshold $r_{s}$ chosen. Finally, not only the computational load demanded by SampEn is notably higher than that of other predictors, but it is also sensitive to the tuning parameters defined above, so their incorrect setting can lead to results that are quite disparate and not easy to interpret. Generally speaking, all the single-lead descriptors $\left(D\left(\mathrm{~V}_{1}\right), \operatorname{RMS}\left(\mathrm{V}_{1}\right), \operatorname{SampEn}\left(L_{s}, r_{s}^{(1)}\right), \operatorname{SampEn}\left(L_{s}, r_{s}^{(2)}\right)\right)$ exhibit weak discriminating capabilities, as confirmed in Table II by their low AUC values.

\section{Multilead Atrial Signal Amplitude Measurement}

The present work has also developed an automatic system of acquisition of the value of the atrial amplitude exploiting information derived from the intrinsic spatial diversity of the multilead ECG (Secs. II-D-II-F). Regarding this multilead perspective, traditional features based on RMS values computed on several leads seem no to be able to characterize fwave amplitude content. Indeed, descriptors derived from the combination of such values cannot depict significant interclass differences, and AUC values in Table II are quite weak too, especially those related to the prediction performance of $\overline{\mathrm{RMS}}_{8}$ and $\overline{\mathrm{RMS}}_{12}$. By contrast, descriptors defined in our PCA-based multilead framework are more robust and reliable than their single-lead counterparts and than classical multilead approaches. The novel predictor $\widetilde{D}_{8}$ succeeds in pointing out significant differences between the two classes of interest, as shown in Table I, which are in keeping with evidence about the predictive power of f-wave amplitude [7]. What is more, CA outcome prediction performance is enhanced by the appropriate use of more than one ECG lead, as we can see in Table II. In Fig. 3 the predictive power of $\widetilde{D}_{L}$ is quantified by the AUC criterion, expressed as a function of the number of ECG leads. The benefits of examining more than one lead are clearly evidenced by the increasing trend of the mean AUC as more leads are progressively introduced into the analysis.

\section{Benefits of PCA-based ECG Signal Approximation}

The second advantage of our multilead approach also pertains to the application of PCA, when compared with the direct examination of AA signal amplitude. From Fig. 3, PCA superiority over the straight computation of f-wave amplitude on the AA signal can be inferred. Indeed, AUC mean values are generally higher than those obtained without PCA preprocessing. In addition, the higher the number of leads, the larger the difference between the two methods, as AUC ranges partially or completely overlap only when $L$ is sufficiently low. Similar conclusions can be drawn from Fig. 4 when examining AUC evolution as a function of the quality of approximation (6). The fewer PCs used in the approximation, the better the prediction performance. PCA typically assigns noisy and/or spurious signal components to the least significant PCs, which seems to enable the extraction of the most meaningful information about atrial amplitude when retaining the dominant PC of the atrial ECG data.

\section{E. ECG-lead Preselection}

This consideration is in line with the increased accuracy in CA outcome prediction when exploiting 8 leads rather than the whole standard ECG. It is commonly acknowledged that over $90 \%$ of the heart's electric activity in physiological conditions can be explained with a dipole source model, whose evaluation can be assessed by measuring its 3 independent components [23]. In principle, two of the limb leads could reflect the frontal plane components, whereas one precordial lead could be chosen for the anterior-posterior contribution. Accordingly, their combination should provide a complete knowledge about the electric heart vector. However, the assumption of a fixedlocation single dipole could be overly simplistic under AF. In fact, the distributed properties of cardiac sources and the effect of the thoracic surface and internal inhomogeneities should also be included in our model. In addition, the precordial leads detect further nondipolar components which are located on the heart frontal plane and have high diagnostic significance [23]. The use of all 12 leads in standard clinical practice is justified by the fact that clinicians can compare the projections of the resultant vectors in 2 orthogonal planes and at different angles, improving pattern recognition. Nevertheless, in our research framework, preselecting the subset of 8 linearly independent leads seems to boost PCA filtering action, as redundant and/or polluting elements are already partially removed before the decomposition, and the most essential and meaningful components are put into evidence more easily. This may explain why $\widetilde{D}_{8}$ outperforms its 12 -lead equivalent.

\section{F. Multilead Atrial Amplitude Computation Modalities}

Further attention should be given to the computation modalities of the multilead descriptor of f-wave amplitude, e.g., how contributions coming from the ECG leads employed are combined with each other. In [21], the mean value of the entries of $\mathbf{D}_{L}$ in eqn. (7) was employed as a predictor (using a different interpolation technique). In this paper, we present an improved version of this algorithm, where the mean is replaced with the median. Both approaches have the common objective of looking for the measure of central tendency of the dataset; roughly speaking, the most recurrent element which can summarize the average information intrinsic to the dataset itself. Experimental evidence reveals that the median value statistic $\widetilde{(\cdot)}$ performs better than the mean $\overline{(\cdot)}$ on our signal database, 
since it is less sensitive to outliers, as mentioned above. Furthermore, the median is more suitable for skewed data distributions, this being often the case of f-wave amplitude, whose visibility and magnitude can depend on lead location. In the light of these considerations, we can interpret CA outcome prediction performance of the features $\bar{D}_{12}$ and $\bar{D}_{8}$ : interclass differences are poorly significant or not significant, so that results presented in [21] do not seem to generalize to the larger dataset used in this work. Finally, the mean value $\bar{D}_{L}$ indistinctly takes contributions from all leads into account. On the contrary, the median $\widetilde{D}_{L}$ exclusively highlights the role played by certain leads in the description of the global trend of f-wave amplitude, while neglecting the extrema of $\mathbf{D}_{L}$, which are generally not representative enough to summarize the main characteristics of this spatial distribution.

\section{G. Lead Subset Selection for Optimal Prediction Performance}

Another interesting result concerns the subsets of leads yielding optimal classification performance based on atrial signal amplitude measures. Table III shows that some lead combinations recur in each subset. In particular, the optimal $L$ lead subset typically includes leads of smaller optimal subsets, together with a new electrode. The presence of leads representing heart electrical activity on multiple planes confirms again the hypothesis that clinical information coming from multiple electrode locations can improve ablation outcome prediction. We can also observe that lead $\mathrm{V}_{1}$ only starts being significant from $L=5$, in contrast with standard medical practice for $\mathrm{AF}$ analysis where $\mathrm{V}_{1}$ is usually the lead of choice [15]. This result can probably be due to the fact that this lead is not close enough to the PVs and the left atrium, thus neglecting significant information from these atrial areas deeply involved in $\mathrm{AF}$ triggering and maintenance.

\section{H. Limitations of the Study}

Our research is hampered by the lack of comparison with endocardial recordings. Multilead surface ECG provides a global overview of heart activity, whereas endocardial signals account for local information. Nevertheless, since we aim at a noninvasive analysis, endocardial recordings were not contemplated in the present study. The superiority of our atrial amplitude measure over conventional CA outcome predictors has been demonstrated. However, its performance in the presence of noise and interference not suppressed by the preprocessing filter remains to be investigated in more detail. Finally, database evaluation is also affected by several confounding factors, e.g., cardioversion and/or drugs, although procedural $\mathrm{AF}$ termination by $\mathrm{CA}$ is not predictive of longterm outcome [24].

\section{CONCLUSiOnS}

This work has proposed a novel procedure for computing f-wave peak-to-peak amplitude to predict short-term CA outcome in persistent AF patients. Our single-lead automatic procedure is based on PCHIP interpolation, and yields numerical results comparable to those reported in previous works [7] while avoiding the shortcomings of manual measures We have also developed an automatic system of acquisition of the atrial amplitude in the multilead ECG based on PCA, which tries to capture and exploit the intrinsic variability of the atrial signal across different leads. The proposed PCA approximation to the multilead atrial signal extracts the most relevant components describing f-wave magnitude spatial distribution over the selected ECG leads. Followed by interpolation and averaging, the algorithm condenses the multilead atrial amplitude into a single descriptor. Results on a database of persistent AF ECGs recorded before ablation have confirmed the ability of this f-wave amplitude descriptor to carry out short-term CA outcome prediction. The proposed multilead approach outperforms single-lead techniques and shows an increased robustness to electrode selection, making it particularly useful in scenarios where some leads do not provide an effective contribution to the recording, e.g., if they get accidentally loose or disconnected from the patient's skin. Further benefits are derived from PCA's data compression capabilities, as signal approximations with decreasing rank progressively improve classification performance by retaining the most descriptive components of the observations while filtering out polluting contributions. We can conclude that the spatio-temporal properties of the surface ECG can quantitatively assess the impact of CA over fibrillatory activity typical of persistent $\mathrm{AF}$ and improve patient selection. Multilead processing of the ECG offers deeper insights into the AA waveform pattern and AF pathophysiology than single-lead methods, as our analysis shows in the context of CA outcome prediction.

\section{REFERENCES}

[1] V. Fuster, L. E. Rydén, D. S. Cannom, and et al. ACC/AHA/ESC 2006 guidelines for the management of patients with atrial fibrillation executive summary. Circulation, 114:700-752, 2006.

[2] H. Calkins, J. Brugada, D. L. Packer, and et al. HRS/EHRA/ECAS expert consensus statement on catheter and surgical ablation of atrial fibrillation: recommendations for personnel, policy, procedures and follow-up. Heart Rhythm, 4(6):816-861, 2007.

[3] M. Haïssaguerre, P. Jaïs, D.C. Shah, and et al. Spontaneous initiation of atrial fibrillation by ectopic beats originating in the pulmonary veins. N. Engl. J. Med., 339:659-65, 1998.

[4] P. Jaïs, M. Haissaguerre, D.C. Shah, and et al. A focal source of atrial fibrillation treated by discrete radiofrequency ablation. Circulation, 95:572-576, 1997.

[5] K. Nademanee, J. McKenzie, E. Kosar, and et al. A new approach for catheter ablation of atrial fibrillation: mapping of the electrophysiologic substrate. J. Am. Coll. Cardiol., 43(11):2044-53, 2004.

[6] M. Haïssaguerre, P. Sanders, M. Hocini, and et al. Changes in atrial fibrillation cycle length and inducibility during catheter ablation and their relation to outcome. Circulation, 109:3007-3013, 2004.

[7] I. Nault, N. Lellouche, S. Matsuo, and et al. Clinical value of fibrillatory wave amplitude on surface ECG in patients with persistent atrial fibrillation. J. Interv. Card. Electrophysiol., 26(1):11-19, Oct. 2009.

[8] A. Van Oosterom, V. Jacquemet, J. M. Vesin, and L. Kappenberger Model-based inferences for the analysis of electrocardiograms during atrial fibrillation. Journal of Electrocardiology, 40:S17-S18, 2007.

[9] A. Van Oosterom. Vectocardiography Based Analysis of Atrial Fibrillation. 36th International Congress on Electrocardiology, Wroclaw, Poland, JAKS Publishing Company, 2009.

[10] L. Sörnmo and P. Laguna. Bioelectrical Signal Processing in Cardiac and Neurological Applications. Elsevier Academic Press, 2005.

[11] B. D. Van Veen and K. M. Buckley. Beamforming: a versatile approach to spatial filtering. IEEE Acoustics, Speech and Signal Processing Magazine, 5(2):4-24, April 1988. 
[12] J. Pan and W. J. Tompkins. A real-time QRS detection algorithm. IEEE Trans. Biomed. Eng., 3(3):230-236, 1985.

[13] A. Cabasson and O. Meste. Time delay estimation: A new insight into the Woody's method. IEEE Signal Processing Letters, 15:573-576, 2008.

[14] D. Kahaner, C. Moler, and S. Nash. Numerical Methods and Software. Prentice Hall, 1998.

[15] S. Petrutiu, J. Ng., G. M. Nijm, and et al. Atrial fibrillation and waveform characterization. IEEE Eng. in Med. and Biol. Mag., 25(6):24-30, 2006.

[16] F. Castells, P. Laguna, L. Sörnmo, A. Bollmann, and J. Millet Roig. Principal component analysis in ECG signal processing. EURASIP Journal on Advances in Signal Processing, 2007:98, 2007.

[17] P. Bonizzi, M. S. Guillem, A. M. Climent, J. Millet, V. Zarzoso, F. Castells, and O. Meste. Noninvasive assessment of the complexity and stationarity of the atrial wavefront patterns during atrial fibrillation. IEEE Trans. Biomed. Eng., 57(9):2147-2157, 2010.

[18] H. L. Van Trees. Detection, Estimation, and Modulation Theory, Part IV: Optimum Array Processing. John Wiley \& Sons, New York, 2002.

[19] J. S. Richman and J.R. Moorman. Physiological time-series analysis using approximate entropy and sample entropy. Am. J. Physiol. Heart Circ. Physiol., 278:2039-2049, 2000.

[20] D.E. Lake, J.S. Richman, M.P. Griffin, and et al. Sample entropy analysis of neonatal heart rate variability. Am. J. Physiol. Heart Circ Physiol., 283:789-797, 2002.

[21] M. Meo, V. Zarzoso, O. Meste, D. G. Latcu, and N. Saoudi. Non-invasive prediction of catheter ablation outcome in persistent atrial fibrillation by exploiting the spatial diversity of surface ECG. In Proc. IEEE EMBS 2011, pages 5531-5534, 2011.

[22] R. Alcaraz and J.J. Rieta. A novel application of sample entropy to the electrocardiogram of atrial fibrillation. Nonlinear Analysis: Real World Applications, 11:1026-1035, 2010.

[23] J. Malmivuo and R. Plonsey. Bioelectromagnetism - Principles and Applications of Bioelectric and Biomagnetic Fields. Oxford University Press, New York, 1995.

[24] C. S. Elayi, L. Di Biase, C. Barrett, and et al. Atrial fibrillation termination as a procedural endpoint during ablation in long-standing persistent atrial fibrillation. Heart Rhythm, 7:1216-1223, Sept. 2010.

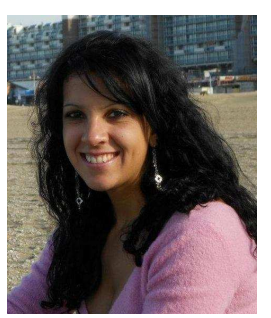

Marianna Meo received the M.Sc. in Biomedical Engineering with full marks from the Campus Biomedico University of Rome in 2009. She is currently working toward the Ph.D. degree at the Computer Science, Signals and System Laboratory of Sophia Antipolis (I3S), University of Nice Sophia Antipolis, France.

Her main research interests concern statistical signal processing, blind source separation and multivariate signal decomposition techniques (principal component analysis, non-negative matrix factorization), clustering and classification, with biomedical applications (atrial fibrillation analysis).

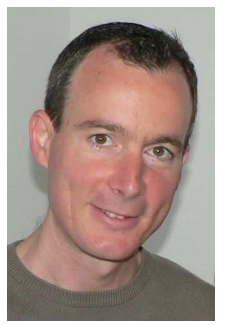

Vicente Zarzoso (S'94-M'03-SM'10) graduated with highest distinction in telecommunications engineering from the Polytechnic University of Valencia, Valencia, Spain, in 1996. He obtained the Ph.D. degree from the University of Liverpool, Liverpool, U.K., in 1999 and the Habilitation to Lead Researches (HDR) from the University of Nice Sophia Antipolis, Sophia Antipolis, France, in 2009.

From 2000 to 2005, he held a Research Fellowship from the Royal Academy of Engineering of the U.K. Since 2005, he has been with the University of Nice Sophia Antipolis, where he is presently a Professeur des universités. His research interests include statistical signal and array processing and its application to biomedical problems and digital communications. He currently coordinates a French National Research Agency Young Investigator's project on array signal processing for atrial fibrillation analysis (ANR "PERSIST").

Dr. Zarzoso is an Associate Editor of the IEEE Transactions on Neural Networks and Learning Systems.

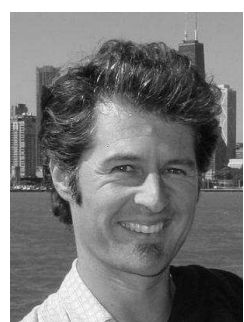

Olivier Meste (M'97) received the M.Sc. degree in electronic engineering and the Ph.D. degree in control and signal processing from the University of Nice Sophia Antipolis, Sophia Antipolis, France, in 1989 and 1992, respectively.

$\mathrm{He}$ is currently a Full Professor at the Computer Science, Signals and Systems Laboratory of Sophia Antipolis (I3S), University of Sophia Antipolis. He heads the Department of Electrical Engineering and Industrial Data Processing at the IUT and the biomedical signal processing research activity at the I3S Laboratory. His current research interests are in the areas of digital processing, time-frequency representations, and modeling of biological signals and systems, including ECG, EMG and EEG.

Dr. Meste is a former member of the Bio Imaging and Signal Processing technical committee of the IEEE Signal Processing Society (2006-2012).

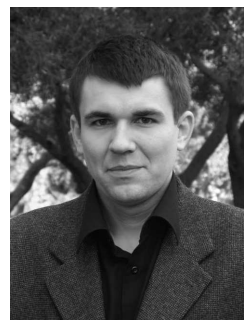

Decebal G. Latcu received the M.D. degree from Carol Davila University, Bucharest, Romania, in 2001. He become a specialist in cardiology from Paul Sabatier University, Toulouse, France, in 2007.

From 2003 to 2007, he was an intern at different hospitals in the Toulouse area. Since 2007, he has been with the Cardiology Department, Princess Grace Hospital, Monaco. His research interests are in the area of cardiac arrhythmias, including ventricular fibrillation, atrial fibrillation and atrial flutter, as well as their computer-aided catheter ablation. Current projects focus on technological developments of atrial fibrillation ablation.

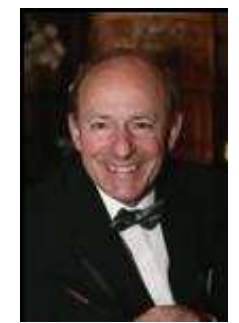

Nadir Saoudi received the M.D. degree from Paris Val de Marne University, Créteil, France, in 1978. He specialized in cardiology from Rouen University Hospital, Rouen, France, in 1984.

From 1978 to 1984 , he was in intern at Fort de France Hospital, Martinique, France, and Rouen University Hospital. From 1984 to 1985, he was a Postdoctoral Research Fellow in cardiac electrophysiology at the University of Miami, FL. In 1993 he become Professor of Cardiology at Rouen University Hospital. He is currently Professor of Cardiology and Head of the Cardiology Department at Princess Grace Hospital, Monaco. His research interests are in the areas of electrocardiology and cardiac arrhythmias. He published the world's first case of catheter ablation of atrial flutter (1985) and the first description of an isthmus block in humans (1995).

Dr. Saoudi is a former President of the French Arrhythmia Group of the French Society of Cardiology, and is also Fellow of the European Society of Cardiology, the Heart Rhythm Society and the American College of Cardiology. He is an Adjunct Professor at the School of Medicine, University of Pennsylvania, PA, and the recipient of a number of awards, including the French Legion of Honor and the Russian Sanitary Merit. 